\title{
Large electron screening effect in different environments
}

\author{
Aleksandra Cvetinović, Matej Lipoglavsek, Sabina Markelu \\ and JELENA VESIĆ
}

Jožef Stefan Institute, Jamova cesta 39, Ljubljana, Slovenia

\begin{abstract}
Electron screening effect was studied on different hydrogen containing targets with the ${ }^{7} \mathrm{Li},{ }^{11} \mathrm{~B}$ and ${ }^{19} \mathrm{~F}$ ion beams. Results show large electron screening potentials strongly dependent on the proton number $Z$ of the projectile. The largest ever measured screening potential with the value about a factor of 50 above the calculations from the model in adiabatic limit was observed in the graphite target containing hydrogen as an impurity.
\end{abstract}

\section{Introduction}

In reactions between nuclei at very low energies, when the energy of the incident beam in the center of mass system is far below the Coulomb barrier, tunneling is the only way the fusion process can happen. But, since the probability for a reaction drops steeply with decreasing energy, the cross sections at thermal energies become extremely difficult to measure. Nowadays, the reactions at these energies can be investigated in underground laboratories with high-current low energy accelerators (such as LUNA [1] and in future DIANA [2]). However, even when astrophysical energies are reached, the measurements do not give the nuclear cross section, since the reaction rate at these energies is strongly influenced by the electrons that surround the reacting nuclei and increase the tunneling probability through the Coulomb barrier. 
The tunneling probability at low energies can be approximated with the Gamow factor $G=e^{-2 \pi \eta}[3]$ which describes the s-wave penetration through the Coulomb barrier of point like charges. Since $G$ strongly depends on the projectile energy, in order to separate the strong energy dependence from effects of pure nuclear interaction, the cross section $\sigma(E)$ is usually presented by introducing the astrophysical $S$-factor, which in the case of non-resonant reactions varies smoothly with energy [3], i.e.:

$$
\sigma(E)=\frac{S(E)}{E} e^{-2 \pi \eta}
$$

Here $\eta=Z_{1} Z_{2} e^{2} / 4 \pi \varepsilon_{0} \hbar \sqrt{2 E / \mu}$ represents the Sommerfeld parameter $\left(Z_{1}\right.$ and $Z_{2}$ are the charge numbers of the interacting nuclei; $E$ is the center of mass energy and $\mu$ is the reduced mass). When the reaction rate is very low, it is sensitive to electronic properties of the target material and the electrons surrounding the reacting nuclei can increase the tunneling probability through the Coulomb barrier, enhancing the cross sections compared to the cross section in the case of the bare nuclei. In order to take into account this effect, an enhancement factor defined as the ratio of screened and bare cross sections [4] is introduced:

$$
f(E)=\frac{\sigma\left(E+U_{e}\right)}{\sigma(E)}
$$

where $U_{e}$ represents the electron screening potential. There are many experimental results published by different groups [5-13] that are inconsistent with the theoretical models (i.e. model in adiabatic limit [4]). Also linear dependence of $U_{e}$ on target $Z$ number was suggested in ref. [10], while findings of our group show a dependence of Ue on target preparation [12, $13]$.

\section{Experiment}

To investigate the electron screening potential, we performed experiments with the ${ }^{1} \mathrm{H}\left({ }^{7} \mathrm{Li}, \alpha\right){ }^{4} \mathrm{He},{ }^{1} \mathrm{H}\left({ }^{11} \mathrm{~B}, \alpha \alpha\right){ }^{4} \mathrm{He}$ and ${ }^{1} \mathrm{H}\left({ }^{19} \mathrm{~F}, \alpha \gamma\right){ }^{16} \mathrm{O}$ reactions in inverse kinematics on physically the same targets. Measurements were performed on five thick proton containing targets. The $50 \mu \mathrm{m}$ thick polyimide (Kapton, $\mathrm{C}_{22} \mathrm{H}_{10} \mathrm{~N}_{2} \mathrm{O}_{5}$ ) foils were used as a reference hydrogen target. The $250 \mu \mathrm{m}$ thick graphite foil was fabricated by Chempur. This target originally contained about $6 \%$ of hydrogen. A small additional 
amount of hydrogen was implanted using our Tectra IonEtch ion gun. The titanium hydride target was pressed from $\mathrm{TiH}_{2}$ powder into a $1 \mathrm{~mm}$ deep hole in a $\mathrm{Cu}$ backing which helped to cool the target after heating with the ion beam. The palladium target was first loaded and unloaded with hydrogen more than 10 times. The cycling was done by leaving the Pd foil in hydrogen gas at 1 bar for 24 hours and then heating in vacuum to $300^{\circ}$ C. After that, the foil was radiation damaged with $45 \cdot 3 \cdot 10^{15}{ }^{19} \mathrm{~F}$ ions per $\mathrm{cm}^{2}$ at $7.8 \mathrm{MeV}$ in order to create lattice defects to which hydrogen could be trapped [14] and then again left in hydrogen gas at 1 bar for another 2 hours. The tungsten target was treated in a similar way. The foil was produced by Plansee and it was mechanically polished and outgassed at $1200 \mathrm{~K}$ for stress relief. Electron microscopy showed large grains $(5-20 \mu \mathrm{m})$ [15]. The foil prepared in this way was pre-damaged with $20 \mathrm{MeV}{ }^{186} \mathrm{~W}$ ions up to radiation damage of 0.45 displacements per atom. Additionally, the foil was implanted with $5 \mathrm{keV}$ protons from the ion gun with a beam intensity of $400 \mu \mathrm{A} / \mathrm{cm}^{2}$ for four hours in steps of half an hour. After each step we performed a reference measurement in order to check for hydrogen concentration. The maximum hydrogen concentration was achieved already after the first half hour. All targets were checked for hydrogen loss by repeatedly measuring yields at the same beam energy before and after each measurement at a given energy. It was found that only the graphite and Kapton targets lost some hydrogen during all measurements, while the $\mathrm{W}$ target lost some hydrogen only in the experiment with ${ }^{19} \mathrm{~F}$ ion beam. In order to correct for the hydrogen loss, the detected yields for a given beam energy were normalized to the average of the two control measurements.

The $\alpha$-particle yield from the ${ }^{1} \mathrm{H}\left({ }^{7} \mathrm{Li}, \alpha\right){ }^{4} \mathrm{He}$ reaction, according to the definition of the cross section $\sigma(E)$ in the case of a thick target, was calculated as [16]:

$$
N_{\alpha}=2 N_{L i} n \frac{\rho N_{A}}{M} \int_{E_{0}}^{0} \varepsilon \omega \frac{\sigma(E)}{d E_{L i} / d x} d E_{L i} .
$$

Here $\varepsilon$ is the efficiency of the detector, $\omega$ is the angular distribution factor of emitted $\alpha$-particles (taken from ref. [17]) and $n$ is the number of hydrogen atoms per crystal lattice atom. The stopping power was calculated using the SRIM code [18]. Electron screening was observed by fitting eq. 3 to experimental data with $U_{e}$ and $n$ as free parameters. The cross sections for bare nuclei $\sigma(E)$, were calculated using equation 2, with the $S$-factor taken from ref. [5]. The $U_{e}$ and $n$ values resulting from least-squares fits to our data together with the predicted values of the electron screening potential 


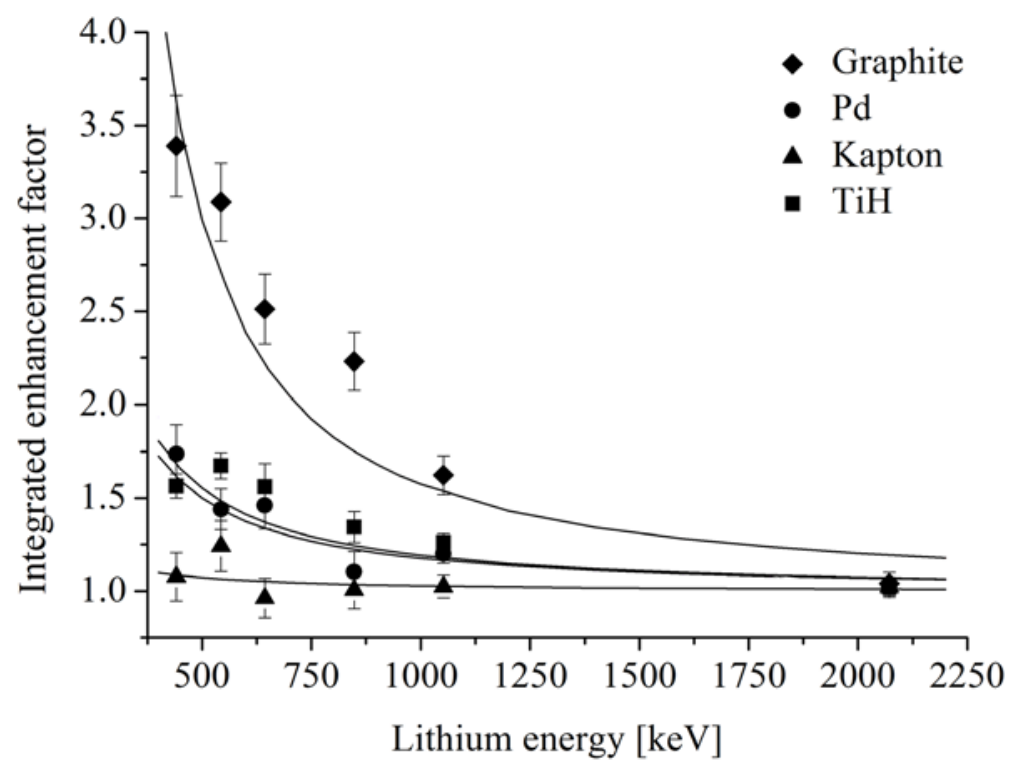

Figure 1: Integrated enhancement factors as a function of lithium beam energy for the ${ }^{1} \mathrm{H}\left({ }^{7} \mathrm{Li}, \alpha\right){ }^{4} \mathrm{He}$ reaction in Kapton, $\mathrm{Pd}$, graphite and TiH targets. The solid lines represent least-squares fits using eq. 3.

calculated from the approximation in adiabatic limit $U_{a d}$ are summarized in table 1. Fig. 1 shows obtained enhancement factors as a function of $\mathrm{Li}$ beam energy in Kapton, $\mathrm{Pd}$, graphite and $\mathrm{TiH}$ targets.

The ${ }^{1} \mathrm{H}\left({ }^{11} \mathrm{~B}, \alpha \alpha\right){ }^{4} \mathrm{He}$ and ${ }^{1} \mathrm{H}\left({ }^{19} \mathrm{~F}, \alpha \gamma\right){ }^{16} \mathrm{O}$ reactions were studied by measuring the narrow resonances at proton energies of $163 \mathrm{keV}$ [19] and $340.46 \mathrm{keV}$ [20], respectively. Since the beams lose all kinetic energy inside of the target region implanted with hydrogen, we observed step-like shaped resonances which are described by the infinitely thick target yield [3]:

$$
Y_{\Delta E \rightarrow \infty}(E)=\frac{\lambda^{2}}{2 \pi} \frac{\omega \gamma}{\varepsilon_{r}^{e f f}}\left[\arctan \left(\frac{E-E_{r}}{\Gamma / 2}\right)+\frac{\pi}{2}\right] .
$$

Here $\varepsilon_{r}^{e f f}$ is the effective stopping power at the resonance energy $E_{r}$, calculated by the SRIM [18] code. Measured $\alpha$-particle and $\gamma$-ray yields we fitted to eq. 4, with $E_{r}, \omega \gamma$ and $\Gamma$ as free parameters. Since $\sigma(E)$ is proportional to $\omega \gamma$, i.e.:

$$
\sigma(E)=\frac{\lambda^{2}}{\pi} \frac{\omega \gamma}{\Gamma}
$$


Table 1: The obtained electron screening potentials in reactions with lithium, boron and fluorine ion beam incident on implanted hydrogen in different targets together with bulk target hydrogen concentrations. The calculated values of the electron screening potentials in the adiabatic limit $U_{a d}[2]$ are also given.

\begin{tabular}{lllll}
\hline Target & $n$ & $U_{e}\left({ }^{7} \mathrm{Li}\right)[\mathrm{keV}]$ & $U_{e}\left({ }^{11} \mathrm{~B}\right)[\mathrm{keV}]$ & $U_{e}\left({ }^{19} \mathrm{~F}\right)[\mathrm{keV}]$ \\
\hline$U_{a d}$ & & 0.24 & 0.68 & 2.19 \\
$\mathrm{TiH}$ & $1.03 \pm 0.04$ & $3.9 \pm 0.4$ & $6.7 \pm 1.8$ & $62 \pm 6$ \\
$\mathrm{Pd}$ & $0.21 \pm 0.03$ & $3.6 \pm 0.7$ & $8.0 \pm 1.9$ & $63 \pm 6$ \\
$\mathrm{~W}$ & $(4.2 \pm 0.2) 10^{-2}$ & $5.9 \pm 0.9$ & - & $74 \pm 15$ \\
$\mathrm{C}$ & $(5.9 \pm 0.3) 10^{-2}$ & $10.3 \pm 0.4$ & $32 \pm 4$ & $115 \pm 8$ \\
\hline
\end{tabular}

and assuming $U_{e}$ Kapton $=0$, the screening potential was calculated according to eq. 2 from the ratio $(\omega \gamma)_{\text {metal }} /(\omega \gamma)_{\text {Kapton. The hydrogen }}$ concentrations we took from the experiment with the ${ }^{7} \mathrm{Li}$ beam since the same targets were used with all three ion beams and since the experiments were performed immediately after each other. For the $\mathrm{W}$ target we observed a surface hydrogen peak in experiments with ${ }^{19} \mathrm{~F}$ (see fig. 2). Since this peak did not result from surface contamination, but from a larger number of hydrogen trapping sites at the surface [21], the $\mathrm{W}$ target was treated as a sum of a thin and a thick target. We assumed that the screening potential on the surface equals the one in the bulk and we found that the surface contains about 4 times higher hydrogen concentration than the bulk. The calculated values of the electron screening potential for the ${ }^{1} \mathrm{H}\left({ }^{11} \mathrm{~B}, \alpha \alpha\right){ }^{4} \mathrm{He}$ and ${ }^{1} \mathrm{H}\left({ }^{19} \mathrm{~F}, \alpha \gamma\right){ }^{16} \mathrm{O}$ reactions are given in table 1 .

As it can be seen from table 1, large electron screening potential was observed in all targets with the highest value observed in graphite target. These results can be interpreted in the following way. The presence of a hydrogen impurity atom in the hexagonal graphite lattice creates a lattice distortion [22] placing the proton always closer to one carbon atom than to the others. This is different from cubic lattice of our metallic targets, where in the undamaged lattice the hydrogen finds an equilibrium position equidistant from the metallic atoms. However, the palladium and tungsten targets were radiation damaged and protons were trapped into crystal lattice vacancies [14], where they are again closer to one metallic atom than to the others. But we observed lower electron screening potentials in $\mathrm{Pd}$ 


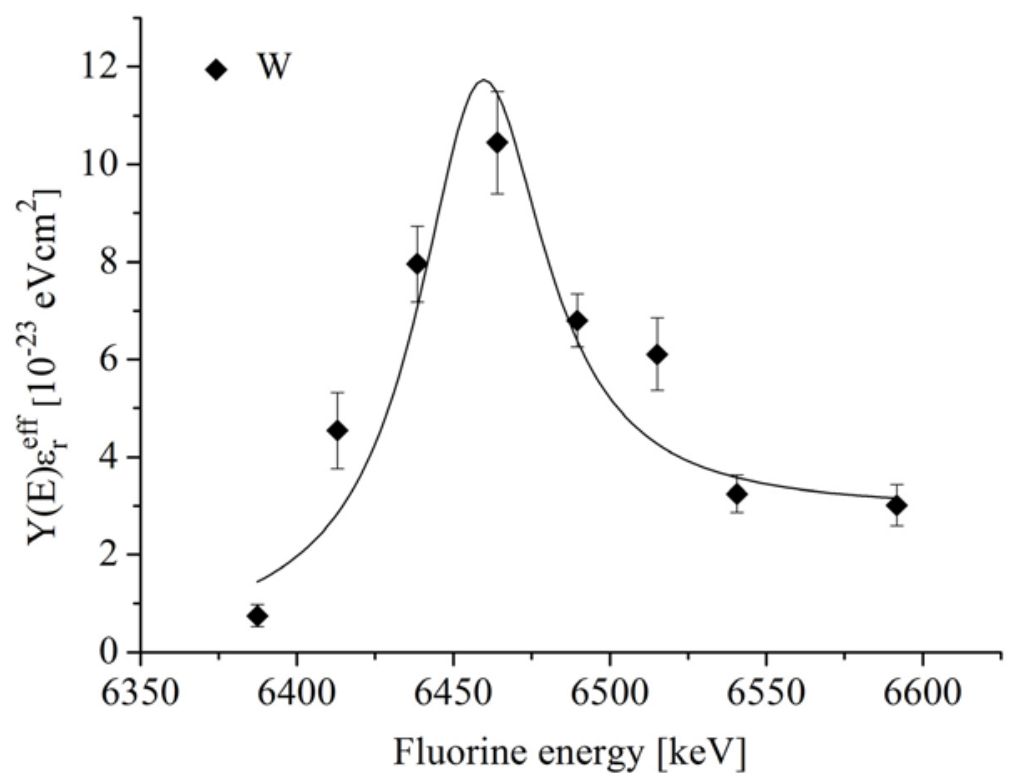

Figure 2: The resonance observed from the ${ }^{1} \mathrm{H}\left({ }^{19} \mathrm{~F}, \alpha \gamma\right){ }^{16} \mathrm{O}$ reaction for $\mathrm{W}$ target fitted to a sum of a thin and an infinitely thick target yields. The $\gamma$-ray yield multiplied by the effective stopping power is shown as a function of beam energy.

and $\mathrm{W}$ targets than in the graphite target. The reason for this is that in polycrystalline metals, hydrogen can also be trapped at grain boundaries and voids [14], where we assume lower electron screening, making the effective electron screening for the two kinds of trapping sites lower. Next, the observed electron screening potential in the Pd target was lower than in the $\mathrm{W}$ target. A possible explanation for this is that our palladium sample was cold rolled, while the tungsten one was annealed. It is known that the cold rolling process increases the number of grain boundary defects, but when the foil is annealed the number of these defects is reduced. Due to this, the resulting effective screening potential in the $\mathrm{Pd}$ target is lower than in $\mathrm{W}$. The $\mathrm{TiH}$ target did not have the full $\mathrm{TiH}_{2}$ stoichiometry, since the powder has been stored in air for several years. The measured stoichiometry of 1.03 means that $\mathrm{TiH}_{1.03}$ is a mixture of fcc and tetragonal fct lattices [23]. As we have shown in earlier experiments with Pd targets [12], the hydrogen on regular interstitial sites in a fcc lattice does not produce a large electron screening effect. Only when the protons are pulled away from their fcc equilibrium positions, a large screening effect occurs. This is the case in the fct lattice in TiH. Finally, from the presented results 
we calculated that the dependence of the electron screening potential on the proton number $Z$ of the projectile seems to be higher than $Z^{2}$.

\section{Summary}

The electron screening effect on thick hydrogen containing graphite, titanium hydride, $\mathrm{Pd}$, and $\mathrm{W}$ targets was investigated by studying the ${ }^{1} \mathrm{H}\left({ }^{7} \mathrm{Li}, \alpha\right){ }^{4} \mathrm{He},{ }^{1} \mathrm{H}\left({ }^{11} \mathrm{~B}, \alpha \alpha\right){ }^{4} \mathrm{He}$ and ${ }^{1} \mathrm{H}\left({ }^{19} \mathrm{~F}, \alpha \gamma\right){ }^{16} \mathrm{O}$ reactions in inverse kinematics. Our measurements are the first ones that studied reactions with different charge numbers using physically the same targets. Contrary to expectations, large electron screening potentials were found in the graphite target: with ${ }^{7} \mathrm{Li}$ beam we observed $U_{e}(L i)=10.3(4) \mathrm{keV}$, with ${ }^{11} \mathrm{~B}$ beam $U_{e}(B)=32(4) \mathrm{keV}$ and $U_{e}(F)=115(8) \mathrm{keV}$ was deduced from the experiment with the ${ }^{19} \mathrm{~F}$ beam. Large electron screening of $U_{e}(L i)=3.9(4)$ $\mathrm{keV}, U_{e}(B)=6.7(18) \mathrm{keV}$ and $U_{e}(F)=62(6) \mathrm{keV}$ was found in titanium hydride. Similarly, high values of Ue were found also in the tungsten target, i.e. $U_{e}(L i)=5.9(9) \mathrm{keV}$ and $U_{e}(F)=74(15) \mathrm{keV}$ and in palladium: $U_{e}(L i)$ $=3.6(7) \mathrm{keV}, U_{e}(B)=8.0(19) \mathrm{keV}$ and $U_{e}(F)=63(6) \mathrm{keV}$.

Also, these results clearly show that the dependence of the electron screening potential on the proton number $Z$ of the projectile is not linear as it was previously supposed, but higher than $Z_{2}$.

\section{References}

[1] D. Bemmerer et al., Eur. Phys. J. A 24, 313 (2005).

[2] D. Leitner et al., Proc. 2011 Particle Accelerator Conf., New York, USA, 2552 (2011).

[3] C. Iliadis, Nuclear Physics of Stars, Wiley-VCH, Weinheim (2007).

[4] H. J. Assenbaum et al., Z. Phys. A 327, 461 (1987).

[5] L. Lamia et al., Astron. Astrophys. A188, 541 (2012).

[6] A. Huke et al., Phys.Rev. C 78, 015803 (2008).

[7] K. Czerski et al., J. Phys. G 35, 014012 (2008).

[8] F. Raiola et al., Eur.Phys.J. A 19, 283 (2004).

[9] J. Kasagi, Prog. Theor. Phys. Suppl. 154, 365 (2004).

[10] J. Cruz et al., J. Phys. G 35, 014004 (2008).

[11] F. Raiola et al., J. Phys. G 31, 1141 (2005).

[12] M. Lipoglavsek et al., Eur. Phys. J. A 44, 71 (2010). 
[13] J. Vesic et al., Eur. Phys. J. A 50, 153 (2014).

[14] Y. Fukai, The Metal Hydrogen System, Springer, Berlin, Germany, (2005) Chapter 5.

[15] O. Ogorodnikova et al., J. Nucl. Mat. 451, 379 (2014).

[16] C. Rolfs and R. W. Kavanagh, Nucl. Phys. A 455, 179 (1986).

[17] S. Engstler et al., Z. Phys. A 342, 471 (1992).

[18] J. F. Ziegler et al., The Stopping and Range of Ions in Matter, Lulu Press Co., Morrisville, NC, (2008), www.srim.org.

[19] Y.S. Chen et al., Nucl. Phys. A 106, 1 (1967).

[20] K. Spyrou et al., Z. Phys. A 357, 283 (1997).

[21] S. Markelj et al., Appl. Surf. Sci. 282, 478 (2013).

[22] C. Herrero and R. Ramirez, J. Phys. D 43, 255402 (2010).

[23] C.P. Liang and H.R. Gong, Mater. Lett. 115, 252 (2014). 